Check for updates

Cite this: RSC Adv., 2018, 8, 30629

\title{
Effects of parathyroid hormone (1-34) on the regulation of the lysyl oxidase family in ovariectomized mice
}

\author{
Linyi Cai, Demao Zhang, Wenjing Liu, Yujia Cui, Junjun Jing, Jing Xie (DD * \\ and Xuedong Zhou*
}

Osteoporosis (OP) is a highly prevalent chronic disease. The anabolic agent parathyroid hormone (PTH) is often prescribed for the treatment of $O P$ to strengthen bone quality and decrease the risk of fracture, although the specific mechanisms are still unclear. Lysyl oxidase (LOX) can stabilize the organic matrix through catalyzing the cross-linking of collagen and elastin. In this study, we established osteoporotic models via ovariectomizing C57BL/6J mice and treating them with PTH. We further aimed to determine the expression changes of the LOX family, impacted by PTH, in ovariectomized mice. We observed that bone mass was reduced and bone microstructure was deteriorative in ovariectomized mice. And PTH attenuated the microstructural damage and accelerated bone remodeling, as confirmed via $\mu \mathrm{CT}$ and $\mathrm{HE}$ staining. Serum levels of copper and zinc indirectly proved the results. The expression levels of five members of the LOX family all declined in ovariectomized mice compared to in sham-operated control mice $(p<0.05)$, and the daily injection of PTH successfully reversed the low expression of LOXs in OP. The current study examined expression changes of LOXs in osteoporotic mice and PTH-treated osteoporotic mice for the first time, and provided an important piece of evidence that the aberrant expression of LOXs had intimate associations with the occurrence and development of OP. And LOXs may act as the downstream effectors of PTH, contributing to unbalanced bone metabolism and damaged bone microstructure. Consequently, LOXs may act as promising therapeutic targets for OP.

\author{
Received 29th May 2018 \\ Accepted 13th August 2018 \\ DOI: $10.1039 / \mathrm{c} 8 \mathrm{ra0} 4574 \mathrm{~g}$ \\ rsc.li/rsc-advances
}

\section{Introduction}

Osteoporosis (OP) is a systematic and disabling bone disease, afflicting mainly elderly postmenopausal women, a consequence of which is that the strength and toughness of bone tissue get reduced and resistance to fracture becomes impaired. ${ }^{1}$ Abnormality of bone quantity and distribution is an important feature of osteoporotic physiopathology. To be specific, cortical bone is manifested as thin and even perforated bone lamellae. As for trabecular bone, progressive decreases in thickness, amount and connectivity can be observed. Moreover, the enhanced formation of adipose tissue, as well as hemopoietic tissue, occurs in bone marrow, increasing the marrow volume. ${ }^{2,3}$ It is generally accepted that these alterations are mainly due to imbalanced bone turnover, and in this process, decreased bone collagen synthesis and increased bone matrix degradation lead to a deteriorating extracellular matrix (ECM). ${ }^{4}$ Despite highly morbidity and mortality, the present understanding of the exact OP pathogenesis is limited, and targeted

State Key Laboratory of Oral Diseases, West China Hospital of Stomatology, Sichuan University, Chengdu, Sichuan 610064, China. E-mail: xiejing2012@scu.edu.cn; zhouxd@scu.edu.cn; Fax: +86-28-85582106; Tel: +86-28-85503465; +86-28-85501481 proteins are sought to provide effective disease-modifying approaches to overcome deficiencies in individuals with OP.

Currently, it is unclear how OP occurs and develops, however bone-resorption inhibitors and anabolic agents have been applied in anti-osteoporotic therapy on the basis of empirical observations. ${ }^{5}$ Bone-resorption inhibitors, also called antiresorptives, preserve bone strength through decreasing bone resorption and suppressing bone turnover. They include bisphosphonates, selective estrogen receptor modulators (SERMs), an inhibitor of receptor activator of nuclear factor- $\kappa \mathrm{B}$ ligand (RANKL), estrogen, and calcitonin. ${ }^{6}$ Among boneforming agents, parathyroid hormone (PTH) is the only one approved by the American Food and Drug Administration (FDA), and it acts as effective medication for OP. ${ }^{4}$ Actually the primary understanding of its function is that it mediates the balance between calcium and phosphorus along with calcitonin in vivo. The targeted organs are kidneys, bones and intestines. ${ }^{7}$ $\mathrm{PTH}$ has a catabolic effect on bone, contributing directly to increased serum calcium and decreased serum phosphorus levels. ${ }^{8}$ As investigations proceeded, its capacity to mediate osteogenesis was found out. Studies have elucidated that PTH administration has therapeutic effects on osteoporotic animal models/patients of normal or low bone mass, ${ }^{\mathbf{9 , 1 0}}$ and intermittent low doses of PTH lead to bone metabolism homeostasis 
being tipped in favor of matrix anabolism, which is the theoretical cornerstone of its clinic application to OP. To date, $\mathrm{PTH}$ has been an efficacious alternative in the treatment of osteoporotic postmenopausal females in clinical settings, but the exact mechanism still remains obscure.$^{\mathbf{1 1}}$

Improving resistance to fracture is the primary goal of OP treatment; this ability is greatly derived from bone toughness, which is supplied primarily by the stability of the bone ECM. ${ }^{12-14}$ Cross-linked collagens, the main components of the bone organic matrix, are critical for stabilizing the polymeric network. ${ }^{15,16}$ It has been evidenced that less mature collagen cross-links are usually accompanied by the deterioration of bone mechanical properties, regardless of whether there are normal mineral quantities or not. ${ }^{17}$ Lysyl oxidase (LOX) plays a significant role in bone collagen biosynthesis and maturation, due to its function of catalyzing cross-linking reactions. More specifically, LOX initiates the covalent cross-linking of elastin and collagen via catalyzing the oxidative deamination of $\varepsilon$-amino groups of lysine and/or hydroxylysine. Ultimately, these extracellular proteins are insolubilized and resist proteolysis. ${ }^{\mathbf{1 8 , 1 9}}$ Except for LOX, the LOX family (LOXs) contains another four members, lysyl oxidase-like protein 1 (LOXL1), lysyl oxidase-like protein 2 (LOXL2), lysyl oxidase-like protein 3 (LOXL3), and lysyl oxidase-like protein 4 (LOXL4). Our previous review has compared the structure of each member and summarized their role in tissue repair. ${ }^{23}$ The five LOX family members show high similarity in their carboxyl (C)-termini, including the copper binding domain, lysyltyrosyl quinone (LTQ) residues, and the cytokine receptorlike (CRL) domain. Besides, the C-terminal domains of LOXs contain the active sites of the enzymes. By comparison, the amino (N)-terminal regions of the LOXs are variable. LOX and LOXL1 are closely related in structure, with both containing a pro-region that is post-translationally processed, and both lack the scavenger receptor cysteine-rich (SRCR) domain present in LOXL2-LOXL4. The distinct sequences of each member may contribute to their different substrates and functions. ${ }^{19}$ The essential roles of LOXs have been recognized in relation to multiple bone disorders. Nagaoka et al. postulated that the directly up-regulated gene expression of LOX could explain the role of $1,25(\mathrm{OH})_{2} \mathrm{D}_{3}$ in many bone defects like rickets, osteomalacia and OP.$^{20} \mathrm{McNerny}$ et al. reported that a reduction of collagen cross-linking and bone ECM synthesis caused by the injection of $\beta$-aminopropionitrile (BAPN), a potent inhibitor of LOX, resulted in destruction of the toughness and strength of bone. ${ }^{21}$ In a study into bone fracture healing, an examination of the transcriptome demonstrated that all five LOXs were highly expressed during the process. ${ }^{22}$ And the variability of the expressions of LOXs is consistent with the alteration of mechanical strength at fractured sites. ${ }^{23}$ In addition, up-regulated LOXL2 was considered a protective response to promote anabolism in cartilage affected by osteoarthritis (OA).$^{24}$

We therefore hypothesized that LOXs may participate in the pathological process of OP. Moreover, PTH could influence the expressions of LOXs under osteoporotic conditions, thus promoting osteogenesis and improving bone microstructure.
To address this issue, we ovariectomized mice to produce an animal model of postmenopausal OP, and examined the effects of PTH on bone metabolism and bone microarchitecture. Most importantly, we examined the expressions of LOXs in vivo, and compared their different expressions under three conditions: sham-operated control conditions, osteoporotic conditions and PTH-treated osteoporotic conditions. To the best of our knowledge, our study is the first to examine the expressions of five LOX family members in mice treated with ovariectomization and PTH. We hope to shed light on the role of LOXs in OP and provide a new targeted protein for use in therapeutic strategies for OP.

\section{Materials and methods}

\section{The development of a mouse OP model and PTH treatment}

Animals were obtained according to ethical principles and protocols were reviewed and approved by our Institutional Review Board (the IRB at the West China Hospital of Stomatology, No. WCHSIRB-D-2017-029), in accordance with national guidelines: Regulations for the Administration of Affairs Concerning Experimental Animals. Two-month-old virgin female C57BL/6J mice of similar body mass were randomly divided into three groups: (1) a sham-operated control group (Control) $(n=$ 12); (2) an ovariectomized group (OVX) $(n=12)$; and (3) a PTHtreated ovariectomized group (OVX $+\mathrm{PTH})(n=12)$, which was ovariectomized and injected with PTH 4 weeks after the operation. Animals had free access to food and water and were housed at $21{ }^{\circ} \mathrm{C}$ under a 12 hours light/dark cycle.

Ovariectomies were conducted as described in a previous paper. ${ }^{3}$ In short, mice were anaesthetized through intraperitoneal injections using sodium pentobarbital (40 $\mathrm{mg} \mathrm{kg} \mathrm{kg}^{-1}$ ) (Sigma-Aldrich, St. Louis, MO, USA). Since the ovaries are located at the midpoint between the lower margin of the free ribs and the iliac crest, we made a lumbar lateral incision around this position. Before removing each ovary, the ovarian artery and vein should be ligated. As for sham operations, ovaries were not resected and we only took out some adipose tissue adjacent to the bilateral ovaries. Finally, muscles were repositioned in layers and sutured with resorbable sutures; the skin was closed with nylon 4-0 sutures. 4 weeks after surgery, mice were treated with daily intraperitoneal injections of either recombinant human PTH (1-34) (40 $\mu \mathrm{g} \mathrm{kg}^{-1}$ ) (Bachem, Bubendorf, Switzerland) or the vehicle (0.9\% sodium chloride, Abbott, IL) for 4 weeks, 6 times a week. All mice were sacrificed after 4 weeks of treatment. We collected blood and knee joint (involving whole femur and tibia samples) specimens for later experiments. 600-800 microlitres of blood from the mouse retro-orbital plexus was collected and centrifuged at $3000 \mathrm{rpm}$ for $10 \mathrm{~min}$ to isolate the serum. The serum samples for the determination of biochemical indicators were frozen at $-80{ }^{\circ} \mathrm{C}$ and stored until measurements were done. Excessive soft tissues were removed from knee joints, then the specimens were fixed in $4 \%$ paraformaldehyde (PFA) for $48 \mathrm{~h}$ at $4{ }^{\circ} \mathrm{C}$ and transferred to $70 \%$ ethanol for longer-term storage until microcomputed tomography ( $\mu \mathrm{CT})$ scanning and histological evaluation was undertaken. 
The evaluation of bone morphometry via micro-computed tomography $(\mu \mathrm{CT})$

To evaluate the alteration of bone structure, knee joints ( $n=$ 12/group) were scanned using $\mu \mathrm{CT}$ apparatus (Y. Cheetah, YXLON International GmbH, Germany). The whole knee joint was set in an immobile fashion inside the scanning tubes, with the respective axes of the tibia and femur as perpendicular as possible to the scanning plane. The parameters were set as follows: voltage, $56 \mathrm{kV}$; current, $61 \mu \mathrm{A}$; and voxel resolution, $0.012 \mathrm{~mm}$. Subsequently, VGS Studio Max software was used to reconstruct the microstructural images. Sagittal and coronal cutting views were used to analyze the structural alteration of trabecular bone. The bone volume fraction (BV/TV) of the femoral diaphysis was also calculated in this way.

\section{Serum levels of biochemical indicators}

Determinations of serum concentrations of copper $(\mathrm{Cu})$ and zinc $(\mathrm{Zn})$ were conducted via atomic absorption spectrometer (AAS) using a PerkinElmer spectrometer. Serum samples and standard working solutions for calibration were prepared via appropriate dilution with deionized water. Standard solutions of $\mathrm{Cu}\left(1000 \mathrm{mg} \mathrm{L}^{-1}\right.$ in $\left.0.3 \mathrm{M} \mathrm{HNO}_{3}\right)$ and $\mathrm{Zn}\left(1000 \mathrm{mg} \mathrm{L}^{-1}\right.$ in $0.5 \mathrm{M} \mathrm{HNO}_{3}$ ) were purchased from the Baker Chemical Co (Phillipsburg, NJ, USA). Except for standard solutions, a blank control was also determined at the same time for quality control. The values of trace elements in the standard solutions were within the ranges provided by the manufacturer.

\section{Histological evaluations}

Mouse knee joints were used for histological evaluations. Firstly, $15 \%$ ethylenediaminetetraacetate (EDTA)-buffered saline solution was used to decalcify samples for 30 days. The samples were bathed in a graded series of ethanol solutions for dehydration and in xylene, and then embedded in paraffin blocks. They were then sectioned into $5 \mu \mathrm{m}$-thick slices. Paraffin-embedded samples were de-paraffinized in xylene, rehydrated using graded alcohol, and immersed in PBS. Slices were stained with H\&E (Sigma-Aldrich, USA) for structural analysis of the bone matrix.

Immunohistochemistry was carried out according to a standard SABC protocol from the manufacturer. De-paraffinized slices were stained with rabbit antibodies against LOXs and goat anti-rabbit IgG/biotin (No. SP-0023; Bioss, Beijing, China). Rabbit monoclonal anti-LOX and anti-LOXL2 were purchased from Abcam (ab174316 and ab179810; Cambridge, MA, USA). Rabbit polyclonal anti-LOXL1 and anti-LOXL4 were purchased from Abcam (ab60178 and ab130646; Cambridge, MA, USA). Rabbit polyclonal anti-LOXL3 was purchased from Santa Cruz (sc-68939; Santa Cruz, CA, USA). The rehydrated samples were pretreated with $0.1 \%$ trypsin for antigen retrieval before staining. All stained slices were scanned with an inverted light microscope (Olympus-IX71, Japan) and analyzed via software (Aperio; Image Scope, USA).

\section{Statistical analysis}

Statistical analysis was performed via the Student's $t$-test and one-way analysis of variance (ANOVA) to determine whether significant differences existed between groups. All data were evaluated via SPSS 20.0 software (SPSS, Chicago, IL, USA) and the critical significance level was set at $p<0.05$.

\section{Results}

\section{The PTH attenuated microstructural damage of trabecular bone contributed to by the ovariectomy}

$\mu \mathrm{CT}$ was employed to elucidate the differences between bone microstructure among the three groups, as it has the ability to assess the quality and quantity of bone. Bone volume and bone structure showed the same substantial changes, determined by $\mu \mathrm{CT}$ analysis: relative to the sham-operated control mice and the PTH-treated ovariectomized mice, the disruption to bone connectivity and microarchitecture in the ovariectomized mice was most remarkable (Fig. 1). Sagittal views of the epiphysis and metaphysis from mouse femora and tibiae showed decreased bone volume in the OVX group (Fig. 1A). The significant bone loss was also exhibited through abnormality in the thickness, amount and continuity of trabecular bone, indicating that disrupted bone remodeling after ovariectomy surgery induced low bone mass accompanied by unsubstantial bone microarchitecture (Fig. 1B). The daily injection of PTH acted to attenuate bone degeneration, manifesting through better connectivity of trabeculae. Additionally, the amelioration of trabecular thickness and amount resulted in a highly organized bone structure in the OVX + PTH group (Fig. 1A and B). In the OP mouse model, the bone volume fraction (BV/TV) of the femoral diaphysis was the lowest, compared to either that of the Control group $(p<0.05)$ or the OVX $+\mathrm{PTH}$ group $(p<0.05)$, and no statistically significant changes were seen between the Control group and the PTH group (Fig. 1C).

\section{PTH improved the ovariectomy-induced homeostatic imbalance of bone metabolism}

Apart from an assessment of general tissue histomorphology, HE staining can also display alterations in bone matrix components, notably trabeculae. So we used HE staining to further prove the mineral changes observed in $\mu \mathrm{CT}$ studies. Fig. 2A shows views of HE staining of tibia and femur samples from the Control group, OVX group and OVX + PTH group. We could see typical bone structure (cortical bone and woven trabeculae, as well as abundant connective tissues), with osteoblasts encapsulated in lacunas of bone matrix and sparsely arranged. The morphology of trabeculae in the Control group was distinct, manifesting as intact and dense. Besides, the existence of fewer inflammatory cells, homogeneously stained ECM, and more ECM deposition suggested stable and relatively slow bone remodeling. In contrast, the boundaries and morphologies of trabeculae in the other two groups were obscure, which was attributed to a discontinuous structure. Furthermore, bone tissue was poorly organized, with numerous inflammatory cells, especially in the OVX group. Significantly, 
A
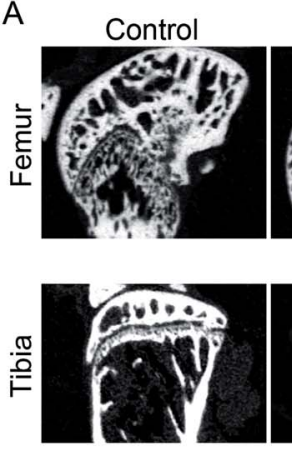

C

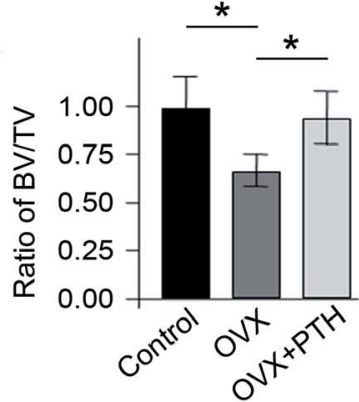

B
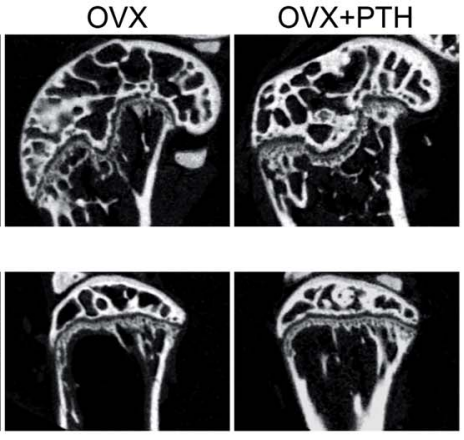

$\overline{550 \mu \mathrm{m}}$
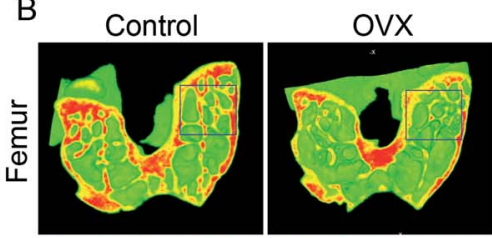

OVX+PTH
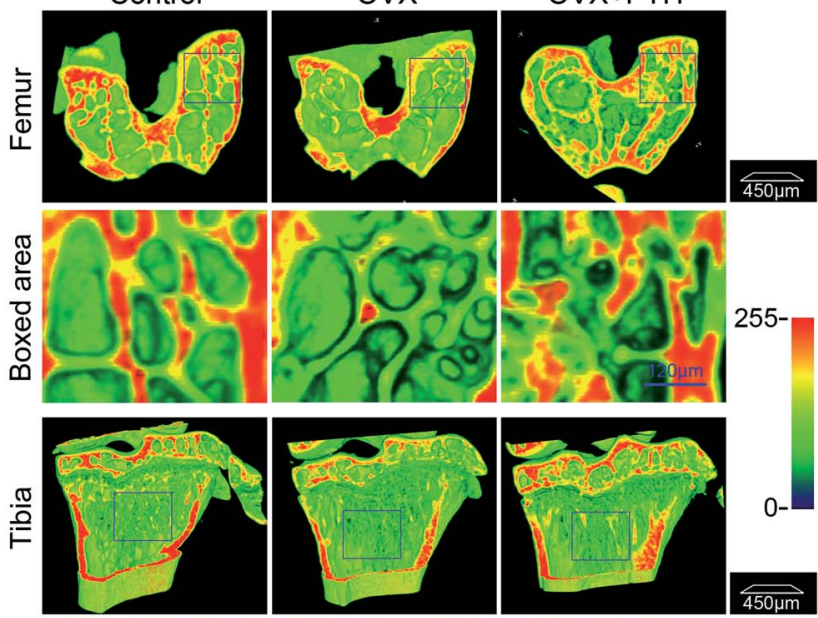

Fig. 1 Changes in bone microstructure in each group evaluated via micro-computed tomography ( $\mu \mathrm{CT}$ ). (A) Top: representative sagittal $\mu \mathrm{CT}$ views of femoral epiphysis and metaphysis samples from 4 months-old control mice (Control), ovariectomized mice (OVX) and PTH-treated ovariectomized littermates (OVX + PTH). Bottom: representative sagittal $\mu \mathrm{CT}$ images of tibial epiphysis and metaphysis samples from mice in the Control group, OVX group and OVX + PTH group. The ovariectomized mice images were collected 2 months after surgery. Scale bar: $550 \mu \mathrm{m}$. (B) Three-dimensional high-resolution $\mu \mathrm{CT}$ images (coronal views) of femoral metaphysis (first two rows) and tibial metaphysis (last two rows) samples. The black rectangles indicate regional magnified images, which are presented in the lower rows. The red areas symbolize higher mineralized tissue, such as cortical bone, and the green parts represent low density tissue, like trabecular bone. Scale bar: $450 \mu \mathrm{m}$. Scale bar in boxed area: $120 \mu \mathrm{m}$. (C) A quantitative analysis of the structural parameters of cortical bone. The bone volume fraction (BV/TV) of the femoral diaphysis indicates significant bone loss appears 2 months after ovariectomy. PTH administration tended to increase the low BV/TV to a level that was comparable to data from the Control group. $n=12$ per group. $* p<0.05$ : statistical differences existed between two groups. All statistical significance was determined via one-way analysis of variance.

bone defects were more prominent in the ovx group, in contrast with the OVX + PTH group in which the progressive transformation of the bone matrix could be observed. So we could predicate that PTH administration effectively resolved bone defects, in spite of the existence of an inflammatory response for the most part. The quantitative analysis of trabecular amounts based on HE staining indicated the intensity of disruption (Fig. 2B). Significant changes were observed between the Control group and the OVX group $(p<0.05)$. And in contrast to the OVX group, the OVX + PTH group presented more trabeculae, owing to the PTH-mediated repair of bone defects $(p<0.05)$.

Considerable disparities in the serum concentrations of $\mathrm{Cu}$ and $\mathrm{Zn}$, the most commonly investigated trace elements in OP, existed between the three groups (Fig. 2C). Ovariectomy resulted in a $43 \%$ decrease in the level of serum $\mathrm{Cu}(p<0.01)$, while PTH treatment could partly reverse the ovariectomyinduced low level of $\mathrm{Cu}(p<0.05)$. There was also a statistical difference in serum $\mathrm{Cu}$ levels between the Control group and the OVX + PTH group ( $p<0.05)$. As for serum Zn levels, no statistical difference existed between the Control group and the OVX group ( $p>0.05$ ). The concentration of serum $\mathrm{Zn}$ was greatly decreased, by $73 \%$, in the OVX + PTH group compared to the Control group $(p<0.01)$. The zinc difference was also significant between the OVX group and the OVX + PTH group $(p<0.01)$. The imaging and biochemical results mentioned above verified the success of the ovariectomy operation and denoted that four weeks of PTH treatment at a dose of $40 \mu \mathrm{g}$ $\mathrm{kg}^{-1} \mathrm{~d}^{-1}$ did have a beneficial effect on bone mass and bone microstructure.

\section{PTH-induced expressions of LOXs in trabecular bone} contribute to improving bone integrity in the situation of $\mathrm{OP}$

To validate the involvement of LOXs in the pathological process of OP, as well as the role of LOXs in the PTH-mediated improvement of bone quality, we applied immunohistochemistry techniques to demonstrate the expression levels of LOX (Fig. 3A and C), LOXL1 (Fig. 4A and C), LOXL2 (Fig. 5A and C), LOXL3 (Fig. 6A and C), and LOXL4 (Fig. 7A and C) in both femoral and tibial trabecular bone. The positive areas of LOXs were significantly less in the OVX group compared to in shamoperated control mice $(p<0.05)$ and PTH-treated ovariectomized mice $(p<0.05)$. Five members, LOX (Fig. 3B and D), LOXL1 (Fig. 4B and D), LOXL2 (Fig. 5B and D), LOXL3 (Fig. 6B and D) and LOXL4 (Fig. 7B and D), clearly showed the same transformation when we calculated relative expressions in the trabecular bone region. Notably, low expressions of LOXs in our 
A
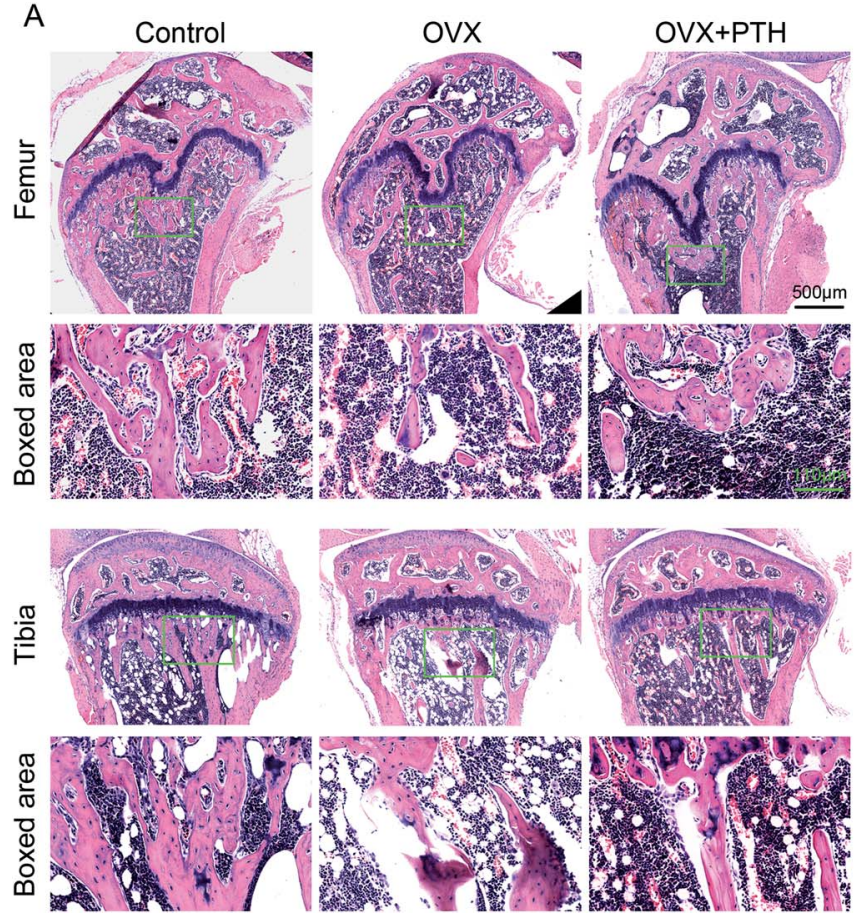

(n)

Fig. 2 Changes in bone histomorphology associated with concentrations of trace elements. (A). HE staining of epiphysis and metaphysis samples of mouse femora (first two rows) and tibiae (last two rows) from control mice (Control), ovariectomized mice (OVX) and PTH-treated ovariectomized littermates (OVX + PTH). The regional magnified images better exhibit the tissue microstructure (green rectangles). Scale bar: $500 \mu \mathrm{m}$. Scale bar in boxed area: $110 \mu \mathrm{m}$. (B). A quantitative analysis of changes in trabeculae amount for femur (left) and tibia (right) samples. $n=$ 12 per group. ${ }^{*} p<0.05,{ }^{* *} p<0.01$ : statistical differences exist between two groups. (C). Concentration changes of copper (Cu${ }^{2+}$, left) and zinc $\left(\mathrm{Zn}^{2+}\right.$, right). $n=12$ per group. $* p<0.05, * * p<0.01$ : statistical differences exist between two groups. All statistical significance values were determined via one-way analysis of variance, and all data are shown as the mean \pm s.d.

mouse OP model were completely reversed through the administration of PTH. And increased levels of LOXs provided protection from the degeneration of the bone matrix, as LOXs were essential for the homeostasis of bone. Therefore, elevated expressions of LOXs in trabecular bone after the daily application of PTH might act as a downstream effector of PTH to suppress aberrant bone metabolism and repair matrix defects. The in vivo results firstly validated the distinct roles of LOXs in OP; high concentrations of active LOXs stimulated by PTH led to the formation of bone matrix and the reconstruction of trabecular bone.

\section{Discussion}

Based on the mouse model of early menopausal OP, this study has evaluated the improvement in osteoporotic condition with the daily administration of PTH from the aspects of bone microstructure, bone metabolism and histological morphology. Notably, through characterizing the differential expressions of LOXs between the Control group, OVX group and OVX + PTH group, we seek to provide important insights into the connection between LOXs and the pathological process of OP, as well as the role of LOXs in the PTH-mediated improvement of bone quality. We found that: (1) PTH improved unbalanced bone metabolism and damaged bone microstructure resulting from ovariectomy; (2) OVX induced lower expressions of LOXs, which

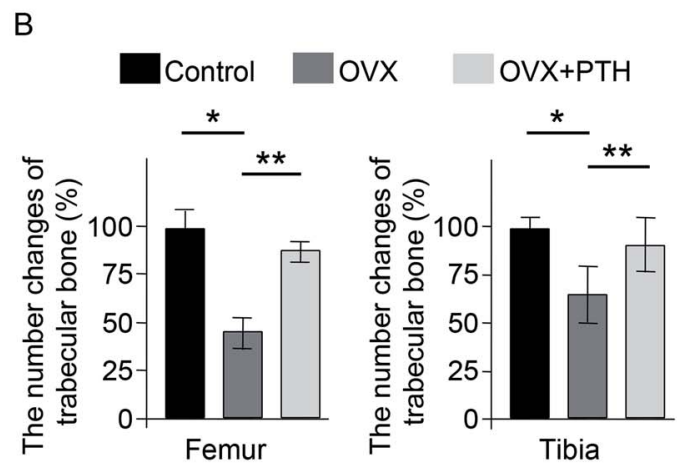

$\mathrm{C}$

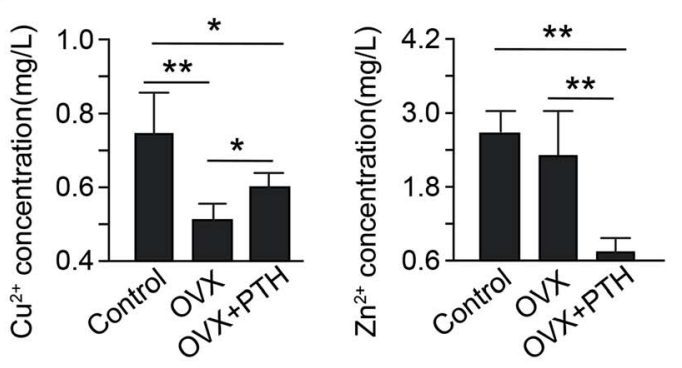

might contribute to the degradation of the bone organic matrix and the progress of OP; (3) PTH treatment could reverse the low expressions of LOXs contributed to by OP, which might explain its promoting effect on bone metabolism and its effectiveness in the treatment of OP. Combined, the up-regulated expressions of LOXs directly promote anabolism after the administration of PTH, and as a consequence, LOXs may act as novel therapeutic targets for OP.

The loss of ovarian hormones during menopause is a major trigger for perturbations in bone remodeling. Subsequently, the decline in bone quantity and quality contributes to deteriorative mechanical and structural integrity, ultimately leading to osteoporotic fractures, especially in those postmenopausal women at high risk for OP. ${ }^{25} \mathrm{Few}$ effective medical therapies for OP have been developed, as its pathogenesis is incompletely illustrated. Antiresorptives are commonly used in the treatment of postmenopausal OP. However, they do not reverse bone fragility, even if the ability to reduce bone remodeling and increase BMD arises during therapy. ${ }^{26}$ The anabolic agent PTH increases bone mass and bone strength via stimulating bone formation and improving trabecular microarchitecture. ${ }^{27}$ It also decreases the risk of both vertebral and non-vertebral fractures, as verified by animal and human trials. ${ }^{28-30}$ Not only can it be used for OP, but PTH can also improve implant osseointegration via increasing the new formation of woven trabeculae in the empty interior implant space. ${ }^{31-33} \mathrm{~A}$ few investigators have reported the 
A
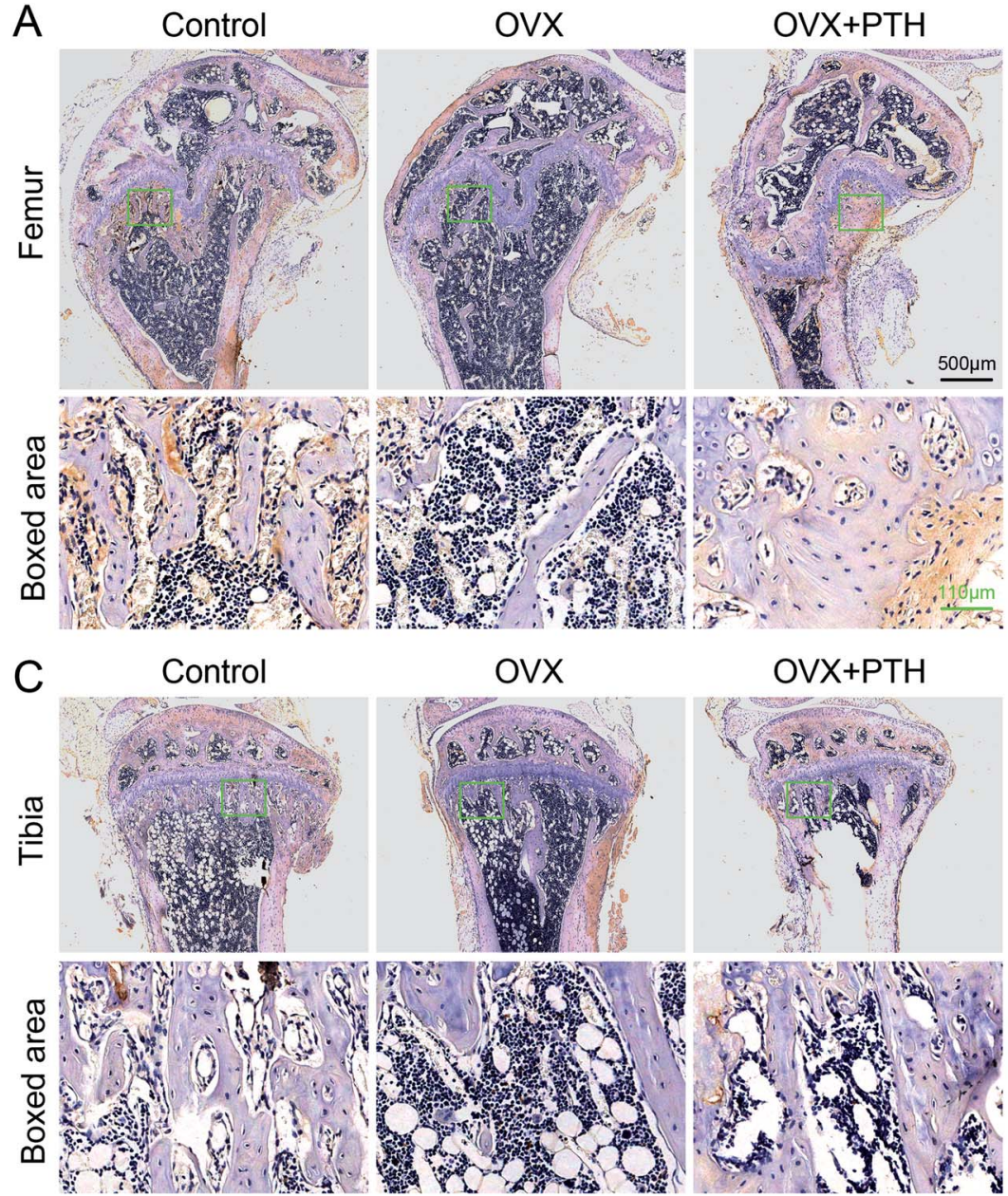

$\mathrm{D}$

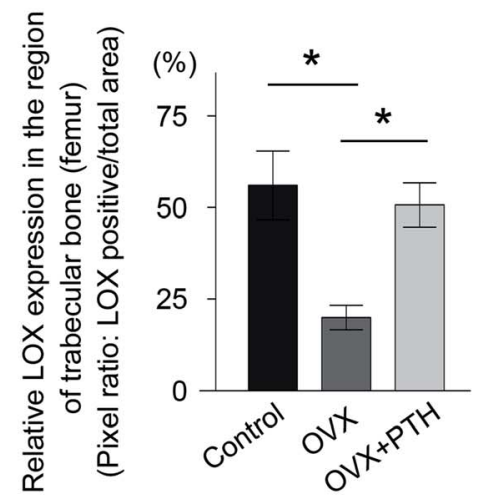

Fig. 3 Immunohistochemistry of LOX in femoral and tibial trabeculae. (A). Top: immunohistochemical staining for LOX in femoral trabeculae from control mice (Control), ovariectomized mice $(\mathrm{OVX})$ and PTH-treated ovariectomized littermates (OVX + PTH). The boxed areas at the site of metaphysis represent regional magnified images, which are shown in the bottom row. Scale bar: $500 \mu \mathrm{m}$. Scale bar in boxed area: $110 \mu \mathrm{m}$. (B). A quantitative analysis of LOX expressed in the region of the femoral trabecular bone. ${ }^{*} p<0.05$ : statistical differences exist between two groups. (C). Top: immunohistochemical staining for LOX in tibial trabeculae from the Control group, OVX group and OVX + PTH group. Boxed areas at the site of metaphysis represent regional magnified images, which are shown in the bottom row. (D). A quantitative analysis of LOX expressed in the region of tibial trabecular bone. * $p<0.05$ : statistical differences exist between two groups. Statistical significance was determined via oneway analysis of variance, and all data are reported as the mean \pm s.d.

mechanism by which the intermittent administration of PTH increases BMD and calcium content. Bellido et al. put forward that PTH resulted in increased osteoblast (OB) numbers and bone mass via attenuating the apoptosis of OBs. ${ }^{34}$ This argument was strengthened by Jilka, who reported that PTH enhanced periosteal and cortical bone formation through antiapoptotic effects on mature OBs and the pro-differentiation of osteoblast lineage cells, while it acted on trabecular bone via anti-apoptotic mechanisms alone in OBs. ${ }^{35}$ Furthermore, it was considered that PTH-mediated survival signaling was selflimiting and required the activation of protein kinase A, the inactivation of the pro-apoptotic protein Bad, and the mediation of cAMP response element-binding protein (CREB) and Runt-related transcription factor 2 (Runx2), as well as the transcription of survival genes like B-cell lymphoma-2 (Bcl-2). The core of the negative feedback was Runx2, because the attenuation of apoptosis depended on induced Runx2. At the same time, PTH also increased Smurf1-mediated proteasomal proteolysis of Runx $2 .{ }^{34}$ In order to further identify the downstream effector proteins of PTH in the treatment of OP, we established an OP mouse model and analyzed the effects over time.

Together with the mineral part, organic components, especially type I collagen, primarily consist of a hierarchical composite - bone ECM. Active remodeling involving a large number of cells (osteoclasts and osteoblasts) perpetually takes place in the bone ECM. It is an orchestrated process in which new structural components take the places of old ones. ${ }^{36,37}$ Most importantly, balanced remodeling is one of the determining factors in bone integrity. Thus it has a strong effect on bone size and shape. Accelerated or unbalanced remodeling contributes to the degradation of the matrix, ultimately leading to bone disorders like OP. OP is characterized by bone loss and a decayed microstructure. Cortical bone becomes thin and 
A
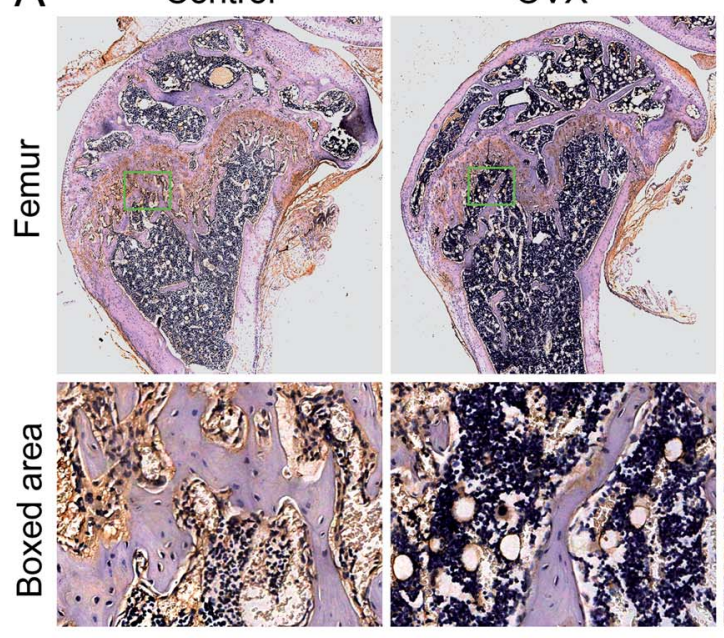

C
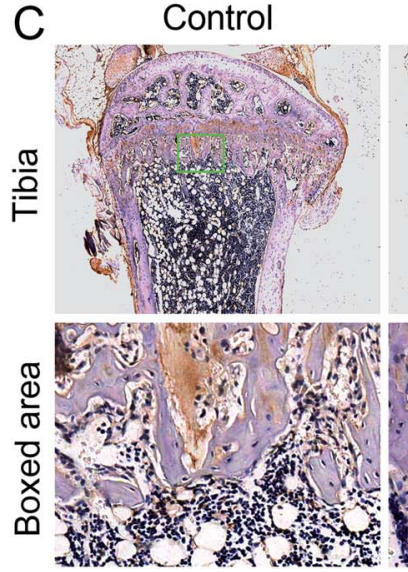
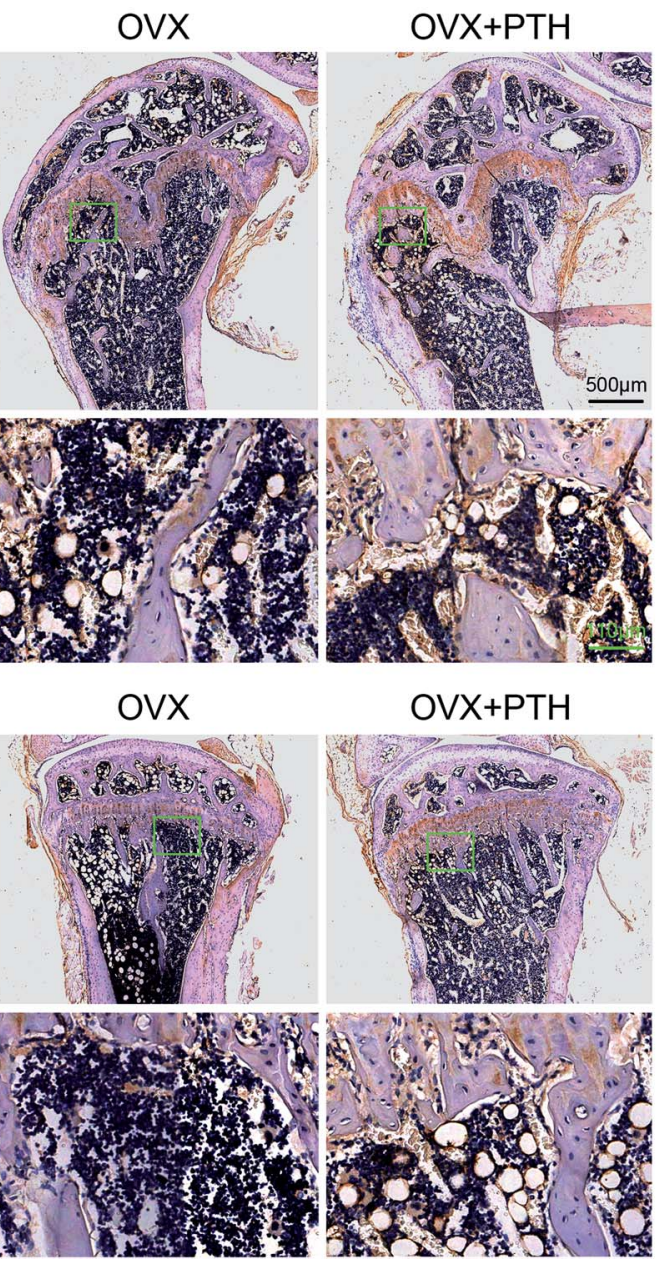

Fig. 4 Immunohistochemistry of LOXL1 in femoral and tibial trabeculae. (A). Top: immunohistochemical staining for LOXL1 in femoral trabeculae from control mice (Control), ovariectomized mice (OVX) and PTH-treated ovariectomized littermates (OVX + PTH). The boxed areas at the site of metaphysis represent regional magnified images, which are shown in the bottom row. Scale bar: $500 \mu \mathrm{m}$. Scale bar in boxed area: $110 \mu \mathrm{m}$. (B). A quantitative analysis of LOXL1 expressed in the region of femoral trabecular bone. ${ }^{*} p<0.05$ : statistical differences exist between two groups. (C). Top: immunohistochemical staining for LOXL1 in tibial trabeculae from the Control group, OVX group and OVX + PTH group. The boxed areas at the site of metaphysis represent regional magnified images, which are shown in the bottom row. (D). A quantitative analysis of LOXL1 expressed in the region of tibial trabecular bone. * $p<0.05$ : statistical differences exist between two groups. Statistical significance was determined via oneway analysis of variance, and all data are reported as the mean \pm s.d.

porous, and trabecular bone becomes disconnected and lost. ${ }^{26}$ In the current study, mouse tibiae and femora were scanned using $\mu \mathrm{CT}$ to evaluated differences in bone mass and bone structure among the three groups. As shown in Fig. 1, images of the epiphysis and metaphysis reveal that OVX induced significant bone loss, and subsequent PTH treatment improved the bone structure from the aspects of the thickness, amount and continuity of trabecular bone. Structural parameters, such as $\mathrm{BV} / \mathrm{TV}$, showed consistent results. Differences in the mineral component were unambiguous among the three different groups, while it has long been understood that osteoporotic bone fragility is not simply attributed to a loss of apatite, in spite of the fact that BMD has been widely employed in the predicting of fractures. The physical properties of bone tissue also rely on the collagen fiber content and biochemistry, ${ }^{38}$ so considering alterations in the organic constituents should not be neglected. In light of the strong association between the quality of the ECM and the resistance of bone to fracture, we thereby focused our attention on variations in the bone matrix with the assistance of HE staining, as it gives a decent exhibition of the quality of matrix components like collagen fibers and trabeculae. ${ }^{39,40}$ The figures indicate that the degradation of collagen and trabeculae, as well as an inflammatory response, was most obvious in the OVX group compared to the other two groups. The results from the OVX + PTH group were comparable to those for the sham-operated control mice. A quantitative analysis of trabecular bone amounts based on HE staining of the femur and tibia further evidenced the trabecular changes observed via $\mu \mathrm{CT}$ analysis.

One of the most important molecular properties of bone collagen is its intermolecular cross-linking. It dominates the pattern in which collagen molecules are arranged in fibrils, and endows fibrillar scaffolds with mechanical properties such as tensile strength and viscoelasticity. Concomitant with 
A

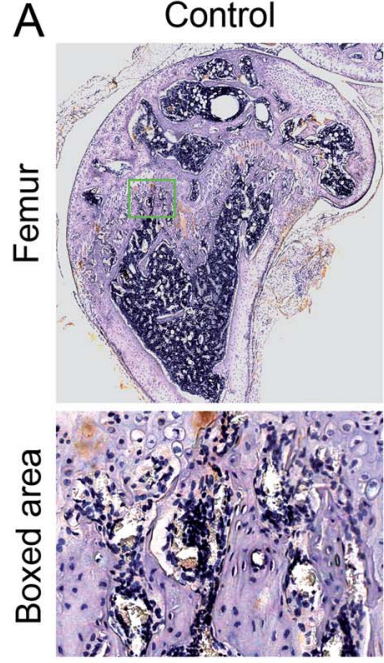

B
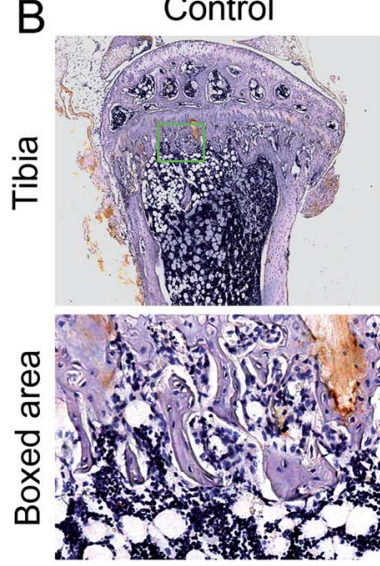

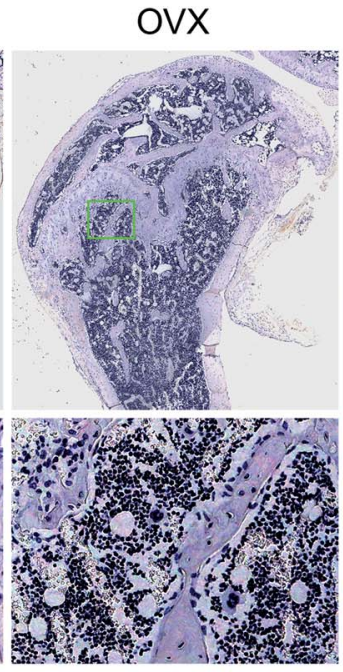

OVX
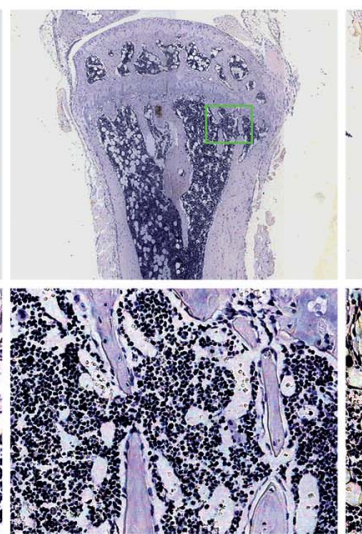

OVX+PTH

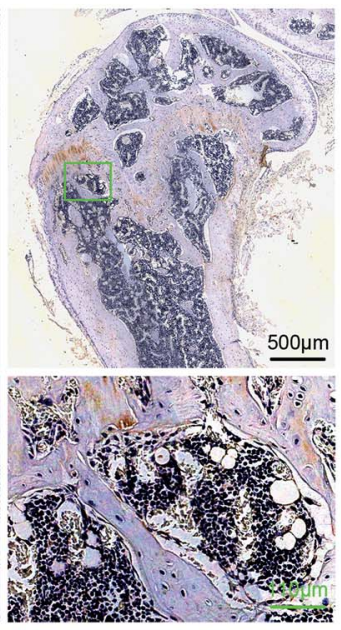

OVX+PTH

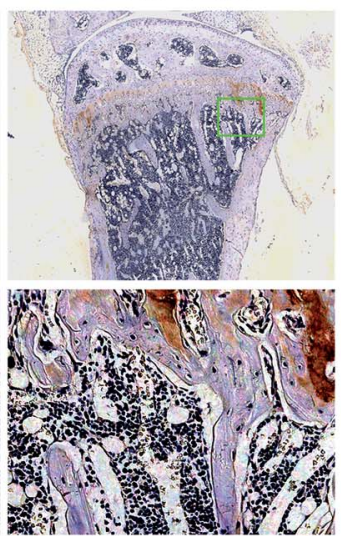

B

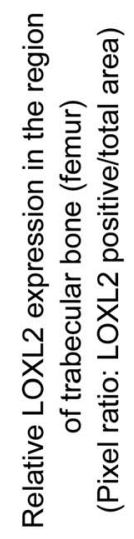

$(\%)$

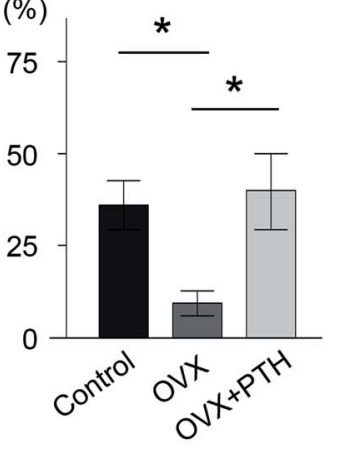

D

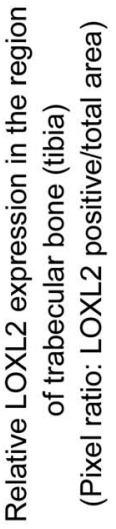

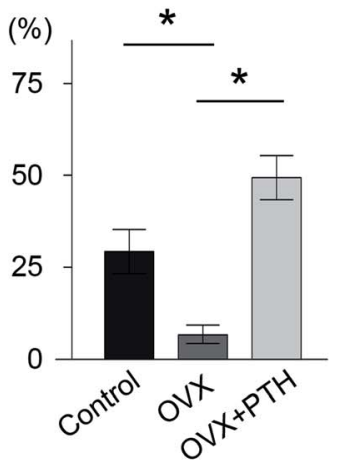

Fig. 5 Immunohistochemistry of LOXL2 in femoral and tibial trabeculae. (A). Top: immunohistochemical staining for LOXL2 in femoral trabeculae from control mice (Control), ovariectomized mice (OVX) and PTH-treated ovariectomized littermates (OVX + PTH). The boxed areas at the site of metaphysis represent regional magnified images, which are shown in the bottom row. Scale bar: $500 \mu \mathrm{m}$. Scale bar in boxed area: $110 \mu \mathrm{m}$. (B). A quantitative analysis of LOXL2 expressed in the region of femoral trabecular bone. ${ }^{*} p<0.05$ : statistical differences exist between two groups. (C). Top: immunohistochemical staining for LOXL2 in tibial trabeculae from the Control group, OVX group and OVX + PTH group. The boxed areas at the site of metaphysis represent regional magnified images, which are shown in the bottom row. (D). A quantitative analysis of LOXL2 expressed in the region of tibial trabecular bone. ${ }^{*} p<0.05$ : statistical differences exist between two groups. Statistical significance was determined via one-way analysis of variance, and all data are reported as the mean \pm s.d.

a decrease in collagen cross-links is a decline in mechanical properties. $^{21,41}$ Mature insoluble cross-links are intimately related to catalysis from LOX and four lysyl oxidase-like proteins. They stabilize the organic matrix through catalyzing the cross-linking reaction of collagen and elastin, thus initiating the formation of covalent cross-links. A body of studies has revealed that aberrant expressions of LOXs could contribute considerably to the abnormal tissue remodeling prevalent in multiple diseases. ${ }^{23}$ Hence, the role of LOXs in preserving bone integrity is self-evident. Generally speaking, we consider the importance of LOXs to bone formation to mainly lie in their catalytic capabilities to promote the maturation of collagen and elastin. From in-depth studies, LOX was found to have other novel functions in the bone matrix. For instance, LOX indirectly promotes cell proliferation through enzymatically modifying growth factor receptors, growth factors, or non-enzymatic functions of the biologically active LOX propeptide. ${ }^{42}$ Under normal physiological conditions, the positive targeting of LOX by Wnt family member $3 \mathrm{~A}$ (Wnt3a) is crucial in maintaining an adequate supply of pluripotent cells, which can differentiate into functional cells. However, while under inflammatory conditions during OP, elevated tumor necrosis factor alpha (TNF- $\alpha$ ) inhibits the up-regulation of LOX mediated by Wnt3a, resulting in a diminished pool of pluripotent cells and ultimately contributing to osteopenia. ${ }^{43}$ We have made a table wherein the role of each member of the LOX family is described (Table 1). Other enzymes participating in the establishment of tissue architecture are matrix metalloproteinases (MMPs). Unlike LOXs, they are a group of endopeptidases capable of degrading collagens and other components of the ECM, in this way directly modulating tissue remodeling and ECM integrity. Dysregulation of MMPs has been generally considered a prominent feature of multiple bone disorders, since considerable research has described the unusual expressions of MMPs 
A

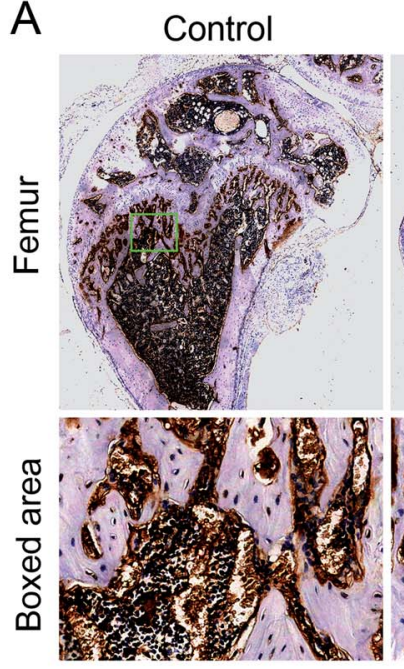

C
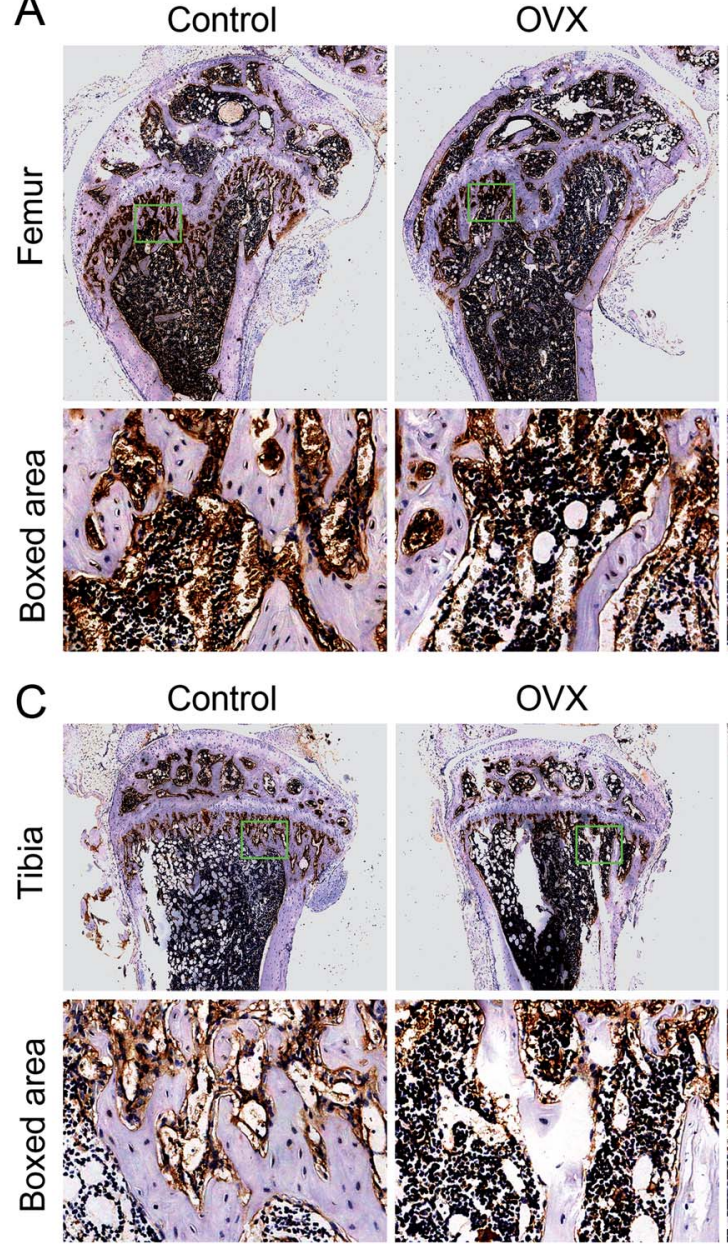

$\mathrm{OVX}+\mathrm{PTH}$

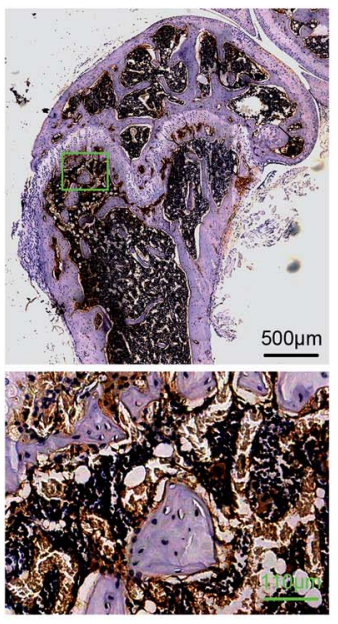

OVX+PTH

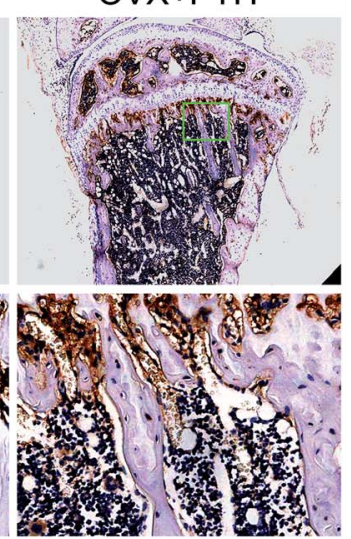

B

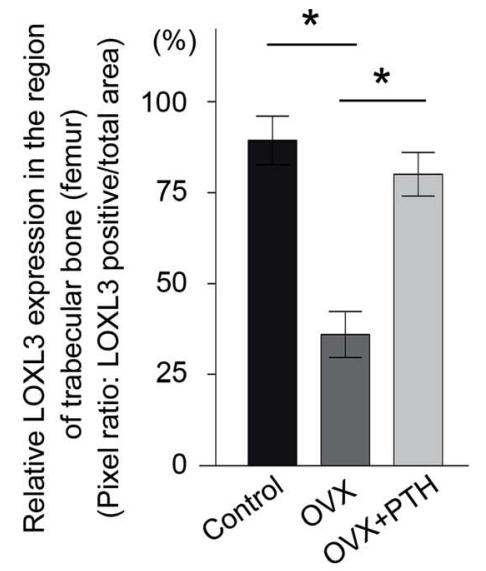

D

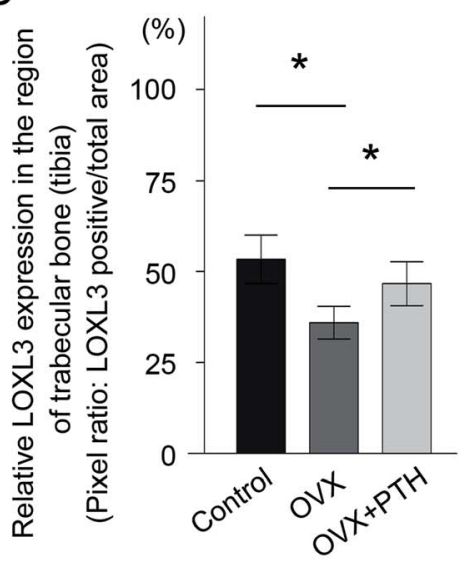

Fig. 6 Immunohistochemistry of LOXL3 in femoral and tibial trabeculae. (A). Top: immunohistochemical staining for LOXL3 in femoral trabeculae from control mice (Control), ovariectomized mice (OVX) and PTH-treated ovariectomized littermates (OVX + PTH). The boxed areas at the site of metaphysis represent regional magnified images, which are shown in the bottom row. Scale bar: $500 \mu \mathrm{m}$. Scale bar in boxed area: $110 \mu \mathrm{m}$. (B). A quantitative analysis of LOXL3 expressed in the region of femoral trabecular bone. * $p<0.05$ : statistical differences exist between two groups. (C). Top: immunohistochemical staining for LOXL3 in tibial trabeculae from the Control group, OVX group and OVX + PTH group. The boxed areas at the site of metaphysis represent regional magnified images, which are shown in the bottom row. (D). A quantitative analysis of LOXL3 expressed in the region of tibial trabecular bone. ${ }^{*} p<0.05$ : statistical differences exist between two groups. Statistical significance was determined via one-way analysis of variance, and all data are reported as the mean \pm s.d.

exactly in the involved regions. ${ }^{44}$ In a study of OA-related cartilage damage, Tchetina found out that MMPs played prominent roles in exacerbation, through immoderately degrading aggrecan and collagen fibers. ${ }^{45,46}$ Destruction of articular cartilage arising from rheumatoid arthritis (RA) was similarly driven by highly expressed MMPs. Given the above, it might be promising to illuminate the pathological mechanism of OP and explore the effector of PTH from the perspective of LOXs.

The main trace elements, such as $\mathrm{Zn}$ and $\mathrm{Cu}$, also correlate with the synthesis of bone collagen. They are responsible for the normal maintenance of bone mass and act mainly as catalysts in synthesizing the organic matrix. ${ }^{47}$ Deficiencies of $\mathrm{Cu}$ and $\mathrm{Zn}$ would retard bone growth, cause fragility, and induce skeleton development disorders, as well as bringing about $\mathrm{OP}{ }^{48}$ As we know, LOXs are $\mathrm{Cu}$-dependent amine oxidases, and the $\mathrm{Cu}$ binding domain is highly homologous in different family members. A study demonstrated that a supplement of $\mathrm{Cu}$ augmented the mediation by LOXs of collagen cross-links through improving activities. ${ }^{49}$ On the other hand, $\mathrm{Cu}$ is associated with decreased bone remodeling, since it suppresses the activities of both OBs and osteoclasts (OCs). ${ }^{50}$ It's also worth mentioning that MMPs are a family of $\mathrm{Zn}$-dependent proteolytic enzymes. Other bone metabolic enzymes, like alkaline phosphatase, sulfuricoylase and calcitonin, are also influenced by $\mathrm{Zn}$. Furthermore, compounds of $\mathrm{Zn}$ and fluorine constitute the bone matrix and have promotive effects on the synthesis of hydroxylapatite. It's also essential for skeletal growth through stimulating the proliferation/differentiation of OBs and diminishing the transformation of OCs. ${ }^{51}$ Although it's a controversial notion that element content values, like those of $\mathrm{Zn}$ and $\mathrm{Cu}$, are strongly associated with BMD, they are the most investigated elements in relation to the development of OP..$^{52}$ We then measured serum levels of $\mathrm{Cu}$ and $\mathrm{Zn}$, hoping to indirectly reflect the transformation of bone mass and the 
A

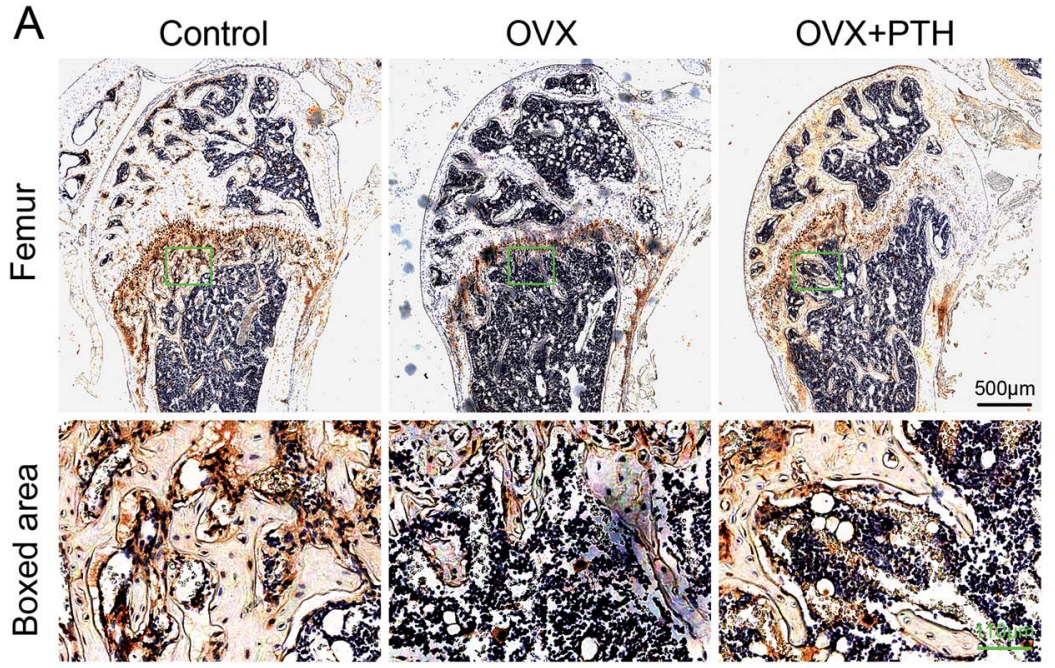

C

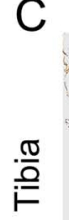

Control<smiles>[131IH]</smiles>
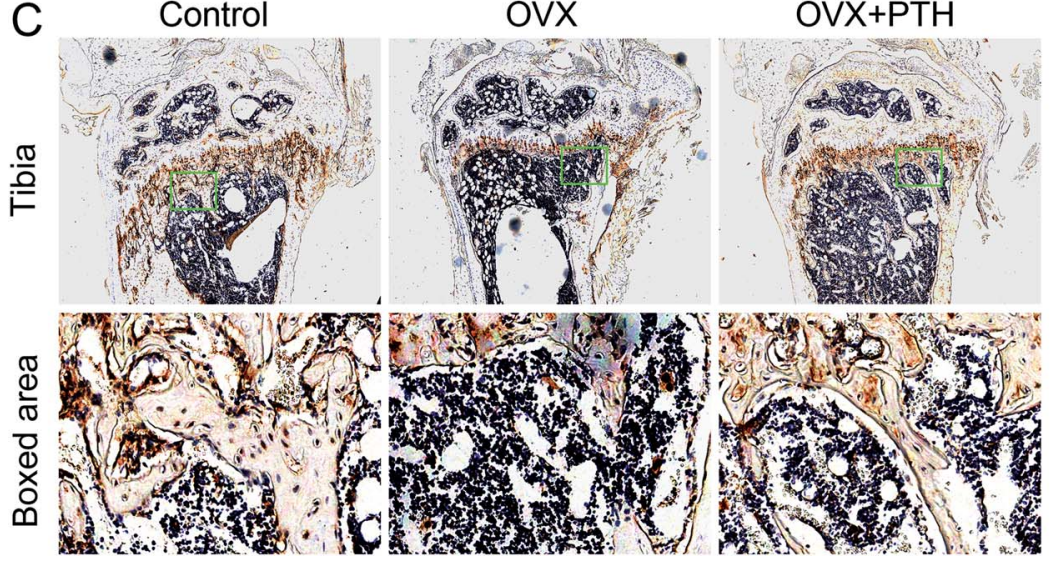

$\mathrm{OVX}+\mathrm{PTH}$

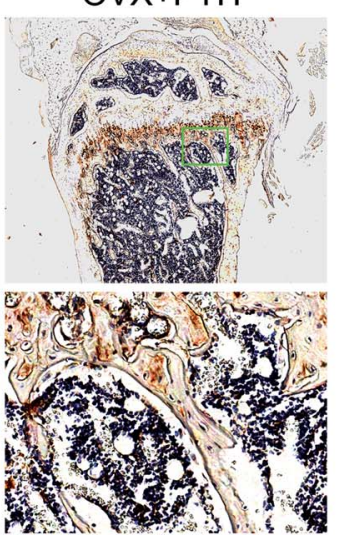

B

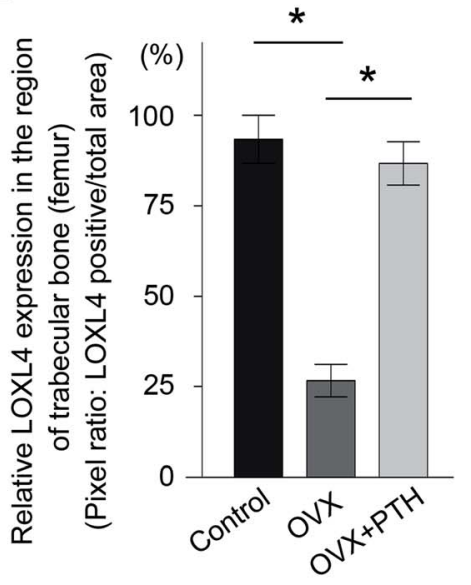

D

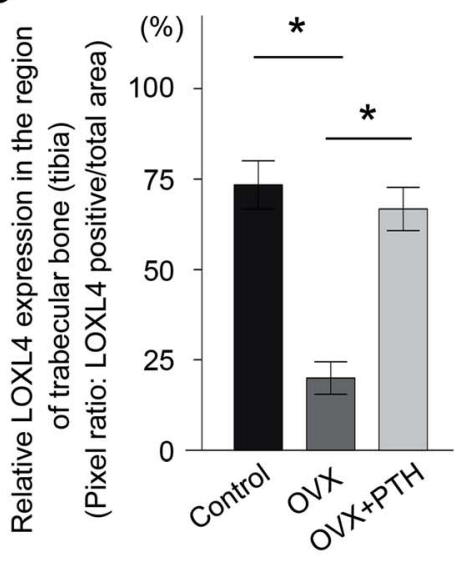

Fig. 7 Immunohistochemistry of LOXL4 in femoral and tibial trabeculae. (A). Top: immunohistochemical staining for LOXL4 in femoral trabeculae from control mice (Control), ovariectomized mice (OVX) and PTH-treated ovariectomized littermates (OVX + PTH). The boxed areas at the site of metaphysis represent regional magnified images, which are shown in the bottom row. Scale bar: $500 \mu \mathrm{m}$. Scale bar in boxed area: $110 \mu \mathrm{m}$. (B). A quantitative analysis of LOXL4 expressed in the region of femoral trabecular bone. $* p<0.05$ : statistical differences exist between two groups. (C). Top: immunohistochemical staining for LOXL4 in tibial trabeculae from the Control group, OVX group and OVX + PTH group. The boxed areas at the site of metaphysis represent regional magnified images, which are shown in the bottom row. (D). A quantitative analysis of LOXL4 expressed in the region of tibial trabecular bone. ${ }^{*} p<0.05$ : statistical differences exist between two groups. Statistical significance was determined via one-way analysis of variance, and all data are reported as the mean \pm s.d.

expression/activity of LOXs and MMPs in each group. OVX resulted in a decreased level of $\mathrm{Cu}$, while the later PTH treatment could increase the level comparative to that of the Control group. The results were exactly in accordance with mineral changes, since bone loss was frequently accompanied by a low level of serum $\mathrm{Cu}$. In addition, a low level of $\mathrm{Cu}$ in our OP mouse model might imply the activities of LOXs decline during OP. PTH administration may improve the condition via induced LOXs. The level of Zn was greatly decreased, by $73 \%$, in the OVX + PTH group in contrast to the OVX group, and we speculated from this finding that there might be a decline in the activities of MMPs after PTH treatment.

Finally, we directly examined the whole expression of LOXs through immunohistochemistry in vivo. In accordance with the variation in serum $\mathrm{Cu}$, we found that the Control group and OVX + PTH group manifested higher expressions of LOXs when comparing with the OVX group. The remarkable reduction of
LOXs validated our hypothesis that LOXs were involved in the pathological process of OP. Another important implication of this work is that up-regulated LOXs may act as effectors of PTH to repair bone defects through stabilizing the organic matrix of bone.

There are several limitations of the current study. Firstly, the daily dose of PTH was $40 \mu \mathrm{g} \mathrm{kg}^{-1} \mathrm{~d}^{-1}$ in the study, which was based on several experiments using the mouse OP model. However, when we explored the correlation between PTH and LOXs, results only from the best dosage of PTH were not so convincing. So different doses of PTH should be applied and then the corresponding expressions of LOXs should be detected in follow-up research. Secondly, expression changes of MMPs and the specific mechanism should be further verified to explain the osteoporotically impaired matrix, together with our results regarding LOXs. Thirdly, ovariectomy does not completely simulate the natural conditions of elderly 
Table 1 The roles of lysyl oxidase family (LOXs) members in bone

Family

member Distribution \& roles

References

LOX Gene knockdown: deficient bone formation in 53,54 vivo; decreased mineral nodule formation and osteoblastic differentiation in vitro; the principal LOX isoform expressed in developing osteoblasts

Osteoblast differentiation: related to collagen $\quad 55,56$ expression/accumulation/deposition, fibril diameter, and BMP-1 expression

Diabetic osteopenia \& diabetes-induced impaired bone healing

Pluripotent mesenchymal cell proliferation and 43 differentiation into osteoblasts

Differentiation of pluripotent mesenchymal $\quad 60$

cells into adipocytes

Participating in the response of PDGF receptors 42,61

to PDGF-BB ligands in smooth muscle cells and megakaryocytes

Regulating bone remodeling by binding to TGF$\beta$ and suppressing its signaling

Bone metastasis: multiple isoforms and multiple domains may have opposing functions, which are only beginning to be understood

LOXL-1 Gene knockdown: a trabecular deficiency in females but hardly any abnormalities in males in vivo; expressed primarily via growth plate chrondrocytes

Diabetic osteopenia

LOXL-2 Gene knockdown: no bone phenotypes have been reported in vivo; deficient expression of early and late chondrocyte differentiation markers in vitro; having tissue-specific distribution in chondrocytes Chondrogenesis during long bone healing \& normal chondrocyte differentiation Inducing angiogenesis and the stabilization of vessels, promoting collagen IV assembly and stabilization

LOXL-3 Gene knockdown: cleft palate, mandibular deformities, and spinal deformities in vivo; expressed primarily via mesenchymal cells in the palate and tongue, and in cartilage

LOXL-4 The expression level is correlated with boneforming capacity in bone marrow progenitor cells

postmenopausal women. This point should not be neglected when comparing the data with clinical results. Fourthly, it is still unclear which of the genes of LOXs is most important for the progress of OP. Since this issue will be of vital importance when designing therapies targeting LOX family members, this obviously needs to be validated in future studies.

\section{Conclusions}

The LOX family stabilizes the organic matrix via catalyzing cross-linking of collagen and elastin. The aim of this study was to evaluate the expression of the different LOX gene family members in long bone samples taken from osteoporotic mice and PTH-treated osteoporotic mice. We found decreased expression levels of each member in the ovariectomized mice compared to the sham-operated control mice, and PTH successfully reversed the low expressions of LOXs under the circumstances of osteoporosis. Thus we conclude that LOXs may act as the downstream effectors of PTH and may act as promising therapeutic targets for osteoporosis.

\section{Conflicts of Interest}

The authors report no conflicts of interest. The authors alone are responsible for the content and writing of the paper.

\section{Acknowledgements}

This work was supported by funding from NSFC grants (81371136, 81430011), and JCPT2011-9 to Xuedong Zhou, funding from NSFC grants (81500876) to Junjun Jing, and funding from NSFC grants $(81600840,81771047)$ to Jing Xie.

\section{References}

1 O. Johnell and J. A. Kanis, Osteoporosis Int., 2006, 17, 17261733.

2 H. Fonseca, D. Moreira-Gonçalves, H. J. Coriolano and J. A. Duarte, Sports Med., 2014, 44, 37-53.

3 Q. Xie, J. Xie, J. Zhong, X. Cun, S. Lin, Y. Lin and X. Cai, Cell Proliferation, 2014, 47, 604-614.

4 A. B. Hodsman, D. C. Bauer, D. W. Dempster, L. Dian, D. A. Hanley, S. T. Harris, D. L. Kendler, M. R. McClung, P. D. Miller, W. P. Olszynski, E. Orwoll and C. K. Yuen, Endocr. Rev., 2005, 26, 688-703.

5 D. Goltzman, Nat. Rev. Drug Discovery, 2002, 1, 784-796.

6 M. P. Ettinger, Arch. Intern. Med., 2003, 163, 2237-2246.

7 C. S. Kovacs, Physiol. Rev., 2014, 4, 1143-1218.

8 D. Miao, B. He, A. C. Karaplis and D. Goltzman, J. Clin. Invest., 2002, 109, 1173-1182.

9 M. Bellido, L. Lugo, S. Castañeda, J. A. Roman-Blas, J. A. Rufián-Henares, M. Navarro-Alarcón, R. Largo and G. Herrero-Beaumont, J. Dent. Res., 2010, 89, 360-365.

10 E. S. Orwoll, W. H. Scheele, S. Paul, S. Adami, U. Syversen, A. Diez-Perez, J. M. Kaufman, A. D. Clancy and G. A. Gaich, J. Bone Miner. Res., 2003, 18, 9-17.

11 M. T. Drake, B. Srinivasan, U. I. Mödder, A. C. Ng, A. H. Undale, M. M. Roforth, J. M. Peterson, L. K. McCready, B. L. Riggs and S. Khosla, Bone, 2011, 49, 349-355.

12 S. Viguet-Carrin, P. Garnero and P. D. Delmas, Osteoporosis Int., 2006, 17, 319-336.

13 X. Wang, X. Shen, X. Li and C. M. Agrawal CM, Bone, 2002, 31, 1-7.

14 K. E. Ensrud and C. J. Crandall, Ann. Intern. Med., 2018, 168, 306-307.

15 S. G. Uzel and M. J. Buehler, J. Mech. Behav. Biomed. Mater., 2011, 4, 153-161.

16 S. P. Robins, Biochem. Soc. Trans., 2007, 35, 849-852.

17 M. G. Orkoula, M. Z. Vardaki and C. G. Kontoyannis, Vib. Spectrosc., 2012, 63, 404-408. 
18 M. Akagawa and K. Suyama, Biochem. Biophys. Res. Commun., 2001, 281, 193-199.

19 H. M. Kagan and W. Li, J. Cell. Biochem., 2003, 88, 660-672.

20 H. Nagaoka, Y. Mochida, P. Atsawasuwan, M. Kaku, T. Kondoh and M. Yamauchi, Biochem. Biophys. Res. Commun., 2008, 377, 674-678.

21 E. M. McNerny, B. Gong, M. D. Morris and D. H. Kohn, J. Bone Miner. Res., 2015, 30, 455-464.

22 M. Bais, J. McLean, P. Sebastiani, M. Young, N. Wigner, T. Smith, D. N. Kotton and T. A. Einhorn, PLoS One, 2009, 4, e5393.

23 L. Cai, X. Xiong, X. L. Kong and J. Xie, Tissue Eng. Regener. Med., 2017, 14, 15-30.

24 W. Alshenibr, M. M. Tashkandi, S. F. Alsaqer, Y. Alkheriji, A. Wise, S. Fulzele, P. Mehra, M. B. Goldring, L. C. Gerstenfeld and M. V. Bais, Arthritis Res. Ther., 2017, 19, 179.

25 M. F. Delaney, Am. J. Obstet. Gynecol., 2006, 194, S12-S23.

26 E. Seeman, J. Gerontol., Ser. A, 2013, 68, 1218-1225.

27 S. Arita, S. Ikeda, A. Sakai, N. Okimoto, S. Akahoshi, M. Nagashima, A. Nishida, M. Ito and T. Nakamura, J. Bone Miner. Metab., 2004, 22, 530-540.

28 S. L. Han and S. L. Wan, Int. J. Clin. Pract., 2012, 66, 199-209. 29 M. H. Murad, M. T. Drake, R. J. Mullan, K. F. Mauck, L. M. Stuart, M. A. Lane, N. O. Abu Elnour, P. J. Erwin, A. Hazem, M. A. Puhan, T. Li and V. M. Montori, J. Clin. Endocrinol. Metab., 2012, 97, 1871-1880.

30 Z. Cheng, W. Yao, E. A. Zimmermann, C. Busse, R. O. Ritchie and N. E. Lane NE, J. Bone Miner. Res., 2009, 24, 209-220.

31 M. I. Almagro, J. A. Roman-Blas, M. Bellido, S. Castañeda, R. Cortez and G. Herrero-Beaumont, Clin. Oral Implants Res., 2013, 24, 1027-1034.

32 Y. Gabet, R. Müller, J. Levy, R. Dimarchi, M. Chorev, I. Bab and D. Kohavi, Bone, 2006, 39, 276-282.

33 Y. Ohkawa, K. Tokunaga and N. Endo, J. Orthop. Sci., 2008, 13, 533-542.

34 T. Bellido, A. A. Ali, L. I. Plotkin, Q. Fu, I. Gubrij, P. K. Roberson, R. S. Weinstein, C. A. O'Brien, S. C. Manolagas and R. L. Jilka, J. Biol. Chem., 2003, 278, 50259-50272.

35 R. L. Jilka, C. A. O'Brien, A. A. Ali, P. K. Roberson, R. S. Weinstein and S. C. Manolagas, Bone, 2009, 44, 275286.

36 D. J. Huey and J. C. Hu, Science, 2012, 338, 917-921.

37 M. A. Karsdal, M. J. Nielsen, J. M. Sand, K. Henriksen, F. Genovese, A. C. Bay-Jensen, V. Smith, J. I. Adamkewicz, C. Christiansen and D. J. Leeming, Assay Drug Dev. Technol., 2013, 11, 70-92.

38 L. Knott, C. C. Whitehead, R. H. Fleming and A. J. Bailey, Biochem. J., 1995, 310, 1045-1051.

39 M. Saito and K. Marumo, Osteoporosis Int., 2010, 21, 195214.

40 J. Y. Chung, M. Song, C. W. Ha, J. A. Kim, C. H. Lee and Y. B. Park, Stem Cell Res. Ther., 2014, 5, 39.

41 E. P. Paschalis, E. Shane, G. Lyritis, G. Skarantavos, R. Mendelsohn and A. L. Boskey, J. Bone Miner. Res., 2004, 19, 2000-2004.
42 H. A. Lucero, K. Ravid, J. L. Grimsby, C. B. Rich, S. J. DiCamillo, J. M. Mäki, J. Myllyharju and H. M. Kagan, J. Biol. Chem., 2008, 283, 24103-24117.

43 R. Khosravi, K. L. Sodek, W. P. Xu, M. V. Bais, D. Saxena, M. Faibish and P. C. Trackman, PLoS One, 2014, 9, e100669. 44 R. Visse and H. Nagase, Circ. Res., 2003, 92, 827-839.

45 E. V. Tchetina, Arthritis, 2011, 2011, 683970.

46 Y. Yoshihara and H. Yamada, Clin. Calcium, 2007, 17, 500508.

47 O. Johnell and J. Kanis, Osteoporosis Int., 2005, 16, S3-S7.

48 M. J. Salgueiro, H. Torti, E. Meseri, R. Weill, J. Orlandini,

R. Urriza, M. Zubillaga, M. Janjetic, A. Barrado and J. Boccio, Biol. Trace Elem. Res., 2006, 110, 73-78.

49 J. E. Marturano, J. D. Arena, Z. A. Schiller, I. Georgakoudi and C. K. Kuo, Proc. Natl. Acad. Sci. U. S. A., 2013, 110, 6370-6375. 50 L. Hoga, J. Rodolpho, B. Gonçalves and B. Quirino, JBI Database System Rev. Implement Rep., 2015, 13, 250-337.

51 E. Okyay, C. Ertugrul, B. Acar, A. R. Sisman, B. Onvural and D. Ozaksoy, Maturitas, 2013, 76, 320-325.

52 S. Z. Liu, H. Yan, P. Xu, J. P. Li, G. H. Zhuang, B. F. Zhu and S. M. Lu, Biol. Trace Elem. Res., 2009, 131, 205-214.

53 R. C. Siegel, Int. Rev. Connect. Tissue Res., 1979, 8, 73-118.

54 N. Pischon, J. M. Maki, P. Weisshaupt, N. Heng, A. H. Palamakumbura, P. N'Guessan, A. Ding, R. Radlanski, H. Renz, T. A. Bronckers, J. Myllyharju, A. M. Kielbassa, B. M. Kleber, J. P. Bernimoulin and P. C. Trackman, Calcif. Tissue Int., 2009, 85, 119-126.

$55 \mathrm{H}$. H. Hong, N. Pischon, R. B. Santana, A. H. Palamakumbura, H. B. Chase, D. Gantz, Y. Guo, M. I. Uzel, D. Ma and P. C. Trackman, J. Cell. Physiol., 2004, 200, 53-62.

56 A. Sharma-Bhandari, S. H. Park, J. Y. Kim, J. Oh and Y. Kim, Int. J. Mol. Med., 2015, 36, 1664-1670.

57 R. Khosravi, K. L. Sodek, M. Faibish and P. C. Trackman, Bone, 2014, 58, 33-41.

58 X. Xiao, J. Ren, J. Chen, Z. Liu, Y. Tian, N. R. Nabar, M. Wang and L. Hao, Biochem. Biophys. Res. Commun., 2018, 495, 821827.

59 R. Khosravi and P. C. Trackman, J. Cell Commun. Signal., 2015, 9, 19-26.

60 H. Huang, T. J. Song, X. Li, L. Hu, Q. He, M. Liu, M. D. Lane and Q. Q. Tang, Proc. Natl. Acad. Sci. U. S. A., 2009, 106, 12670-12675.

61 A. Eliades, N. Papadantonakis, A. Bhupatiraju, K. A. Burridge, H. A. Johnston-Cox, A. R. Migliaccio, J. D. Crispino, H. A. Lucero, P. C. Trackman and K. Ravid, J. Biol. Chem., 2011, 286, 27630-27638.

62 P. Atsawasuwan, Y. Mochida, M. Katafuchi, M. Kaku, K. S. Fong, K. Csiszar and M. Yamauchi, J. Biol. Chem., 2008, 283, 34229-34240.

63 T. R. Cox, R. M. H. Rumney, E. M. Schoof, L. Perryman, A. M. Hoye, A. Agrawal, D. Bird, N. A. Latif, H. Forrest, H. R. Evans, I. D. Huggins, G. Lang, R. Linding, A. Gartland and J. T. Erler, Nature, 2015, 522, 106-110.

64 L. Alsofi, E. Daley, I. Hornstra, E. F. Morgan, Z. D. Mason, J. F. Acevedo, R. A. Word, L. C. Gerstenfeld and P. C. Trackman, Calcif. Tissue Int., 2016, 98, 172-185. 
65 M. Iftikhar, P. Hurtado, M. V. Bais, N. Wigner, D. N. Stephens, L. C. Gerstenfeld and P. C. Trackman, J. Biol. Chem., 2011, 286, 909-918.

66 A. Martin, F. Salvador, G. Moreno-Bueno, A. Floristan, C. Ruiz-Herguido, E. P. Cuevas, S. Morales, V. Santos, K. Csiszar, P. Dubus, J. J. Haigh, A. Bigas, F. Portillo and A. Cano, EMBO J., 2015, 34, 1090-1109.

67 M. Bignon, C. Pichol-Thievend, J. Hardouin, M. Malbouyres, N. Brechot, L. Nasciutti, A. Barret, J. Teillon, E. Guillon,
E. Etienne, M. Caron, R. Joubert-Caron, C. Monnot, F. Ruggiero, L. Muller and S. Germain, Blood, 2011, 118, 3979-3989.

68 J. Zhang, R. Yang, Z. Liu, C. Hou, W. Zong, A. Zhang, X. Sun and J. Gao, Hum. Mol. Genet., 2015, 24, 6174-6185.

69 K. H. Larsen, C. M. Frederiksen, J. S. Burns, B. M. Abdallah and M. Kassem, J. Bone Miner. Res., 2010, 25, 796-808. 